\title{
Numerical Self-Propulsion Assessment of a Generic Submarine Model at Various Forward Speeds
}

\author{
(D) Taner Çoşgun \\ Ylldız Technical University, Department of Naval Architecture and Marine Engineering, İstanbul, Turkey
}

\begin{abstract}
In this study, we use computational fluid dynamics (CFD) to investigate the self-propulsion characteristics of a submarine model. Predicting a marine vehicle's self-propulsion features, and as a result, determining the thrust force required to drive the ship with a constant forward speed is critical for the propulsive system and main engine selection. A Reynolds-Averaged-Navier-Stokes Equations based numerical methodology has been applied to the flow field around the Defense Advanced Research Projects Agency suboff geometry to predict the selfproportion characteristics of a marine vehicle. First, the model's self-propulsion characteristics were determined for a relatively lower hull speed (5.35 knots), and the results were compared with those of other studies and experiments. The study was then extended to include higher forward speeds ranging from 5.93 to 17.79 knots. The results reveal that the propeller rotation rate at the model's self-propulsion point rises as the vessel speed and the power requirement increase. Similarly, the advance coefficient remains nearly unaffected by the Froude Number. The resistance components, propulsion characteristics, flow field surrounding the model, and the wake structure in the propeller slipstream were also evaluated for the determined self-propulsion points.
\end{abstract}

Keywords

Self-propulsion estimation, DARPA, Submarine, Resistance, Computational fluid dynamics

\section{Introduction}

Determining the propulsion performance is one of the key features of the initial design stage of marine vehicles. As the performance of a propeller behind a hull generally differs from that of open water tests, self-propulsion assessments provide valuable information in ensuring that a ship equipped with the propeller can operate with the requisite forward speed. Therefore, in the recent decade, numerous research efforts have been focused on the high-fidelity selfpropulsion predictions of surface ships and submarines [1-5]:

The conventional method for evaluating the hydrodynamic performance of marine vehicles is to conduct model testing. Although model tests produce reliable data, they are time-consuming and expensive. Computational fluid dynamics (CFD) has been gaining attention as an effective and reliable alternative tool for investigating the hydrodynamics and flow details around floating bodies. For self-propulsion investigations and other fields of numerical marine hydrodynamics, numerical simulations, such as virtual towing tank tests, have become a common technique. Carrica et al. [6] numerically presented a method for predicting the self-propulsion point of three benchmark ship geometries. They aimed at satisfying the thrust-resistance equilibrium by controlling the propeller rotational speed. Using CFD calculations, Castro et al. [7] predicted the full-scale self-propulsion computations of the KRISO Container Ship (KCS) hull. They concluded that the propeller operates more efficiently in full scale than in model scale calculations. Shen et al. [8] investigated the self-propulsion and maneuvering of KCS using the opensource CFD tool OpenFOAM with the dynamic overset grid technique. Gaggero et al. [9] considered the same KCS test case to apply the coupled Boundary Element

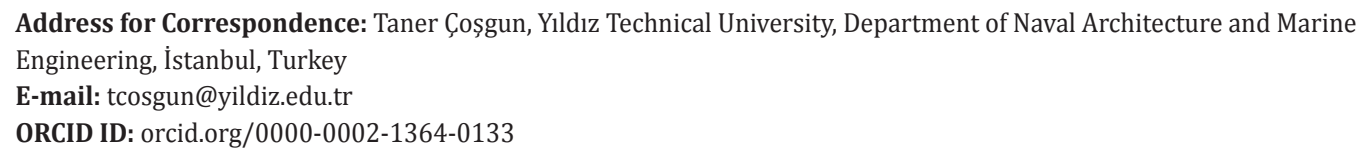

To cite this article: T. Coşgun, "Numerical Self-Propulsion Assessment of a Generic Submarine Model at Various Forward Speeds." Journal of ETA Maritime Science, vol. 9(3), pp. 192-199, 2021.

${ }^{\circ}$ Copyright 2021 by the Journal of ETA Maritime Science published by UCTEA Chamber of Marine Engineers 
Method/Reynolds Averaged Navier-Stokes (RANS) approach to obtain self-propulsion characteristics. He et al. [10] performed a gradient-based design optimization of self-propulsion for a Japanese bulk carrier (JBC). Their study focused on using the proposed method that considers the hull-propeller interaction and allows the use of a large number of design variables to optimize the shape of the stern region of the hull. Bakica et al. [11] used OpenFOAM to calculate the self-propulsion characteristics of the JBC hull and investigate the wake field of the KCS hull. Feng et al. [12] proposed a new body force method coupled with the blade element momentum theory to estimate the self-propulsion performance of the KCS hull. They reported that while preserving the body force method's computational efficiency, the new model considers the three-dimensional viscous effects to improve the fidelity of the predictions. Sezen et al. [13] investigated the self-propulsion characteristics of a full-scale vessel. They also examined the scale impact on the results and put the 1978 International Towing Tank Conference (ITTC) performance prediction method to the test. Several researchers have also studied the self-propulsion performance of ships at full scale [14-16].

Predicting the propulsion characteristics of submarines, like surface ships, has been the subject of several studies. Chase [17] developed an inhouse CFD solver to investigate the effects of the turbulence models on the self-propulsion performance and wake field of the Defense Advanced Research Projects Agency (DARPA) submarine model. Zhang and Zhang [18] investigated the self-propulsion characteristics and resistance of a submarine model operating close to a free surface. Sezen et al. [19] applied RANS based numerical methodology on analyzing a benchmark submarine's self-propulsion performance. They compared the body force (actuator disc) method's results with the self-propelled submarine model calculations. Kinaci et al. [20] estimated a submarine's self-propulsion points and two surface ship models. They also performed the same calculations using a classical engineering approach in addition to the CFD analysis. They compared the results of different methodologies with those of other researchers. Carrica et al. [21] investigated the self-propulsion characteristics of a generic Joubert BB2 submarine model in free surface proximity conditions. Their study also included waves' effect on the propulsion performance and wake in the propeller slipstream.

In this paper, we discuss the numerical estimation of the self-propulsion characteristics of the DARPA submarine model. A RANSE based commercial finite volume solver,
Siemens Star CCM+, was used in the computations. Selfpropulsion features of the model were calculated at a relatively lower forward speed first to compare and validate the present results with other studies. The study was then extended to include higher hull velocities. The submarine model's self-propulsion points and power requirements were calculated for various forward speeds. Furthermore, both self-propelled and towed cases were subjected to resistance analysis. Limited wake stricter investigations were presented to understand the change in the resistance components.

\section{Materials and Methods}

\subsection{Numerical Modeling}

This section presents the numerical modeling details. Below are the descriptions of the benchmark submarine model's geometrical features, numerical methodology, and grid topology.

\subsection{Geometry of the Problem}

The computations are conducted on the benchmark submarine model introduced by DARPA. The DARPA's submarine form had two configurations at first: AFF-1 and AFF-8. The fundamental distinction between both forms is that AFF- 8 has appendages like rudders and sail, whereas AFF-1 is a bare hull form with no appendages. For the current numerical simulations, the AFF-8 submarine hull form shown in Figure 1 is selected. Table 1 lists the main dimensions of the model. Ref. [22] and [23] provide more details about the AFF-8 submarine form. The self-propulsion computations used an INSEAN E1619 propeller. The propeller has seven blades and is $0.262 \mathrm{~m}$ in diameters. The propeller's details can be found in [24].

Table 1. The main properties of the DARPA submarine model

\begin{tabular}{|c|c|}
\hline Parameter & Dimension \\
\hline$L_{O A}(m)$ & 4.356 \\
\hline$L_{B P}(m)$ & 4.261 \\
\hline$D_{\max }(m)$ & 0.508 \\
\hline$S\left(m^{2}\right)$ & 6.348 \\
\hline$\nabla\left(m^{3}\right)$ & 0.706 \\
\hline \multicolumn{2}{|c|}{ DARPA: Defense Advanced Research Projects Agency } \\
\hline
\end{tabular}

\subsection{Governing Equations and Solution Strategy}

The velocity and pressure fields were obtained using a numerical solution of the time-dependent Navier-Stokes equations and the continuity equation. The equations 1 and 2 are:

$$
\frac{\partial u_{i}}{\partial x_{i}}=0
$$


where $u_{i}$ is the time-averaged velocity,pis the pressure, $\rho$ is the density, and $\mu$ is the dynamic viscosity. The last term on the right-hand side of Eq. 2 denotes the Reynolds stress tensor, representing the turbulence effects on the mean momentum.

$$
\rho\left(\frac{\partial u_{i}}{\partial t}+u_{j} \frac{\partial u_{i}}{\partial x_{j}}\right)=-\frac{\partial p}{\partial x_{i}}+\frac{\partial}{\partial x_{j}}\left(\mu \frac{\partial u_{i}}{\partial x_{j}}-\rho \overline{u_{i}^{\prime} u_{j}^{\prime}}\right)
$$

A commercial CFD tool, Star $\mathrm{CCM}+$, is used in the computations. The solver implements the finite volume method for discretizing the governing equations. To improve the accuracy of the solution, a temporal and spatial discretization was done using a second-order scheme. The SIMPLE algorithm is used for velocity-pressure coupling.

The turbulent field is modeled using the realizable k-e turbulence model with the wall-function approach. The turbulence model is described in detail in the solver's documentation [25].

The rotational motion of the propeller was modeled using the rigid body motion (RBM) method, often known as the sliding interface technique. Simulations were first initialized steadily with the moving reference frame method, which simulates the quasi-steady flow around the propeller. After the steady simulations had converged, the numerical procedure was switched to unsteady. Here, the aim is to provide preliminary data for the unsteady simulations.

\subsection{Computational Domain and Boundary Conditions}

The flow around the submarine model was solved using a rectangular-shaped computational domain. Numerical predictions were performed in a Cartesian coordinate system with the negative $\mathrm{x}$-axis in the incoming flow direction and the positive +z-axis pointing upwards. The origin of the coordinate system was located in the submarine model's aft peak. The submarine model was placed at the 2LBP and 5LBP, away from the inlet and outlet boundaries. The solution domain's sidewalls were extended to a length
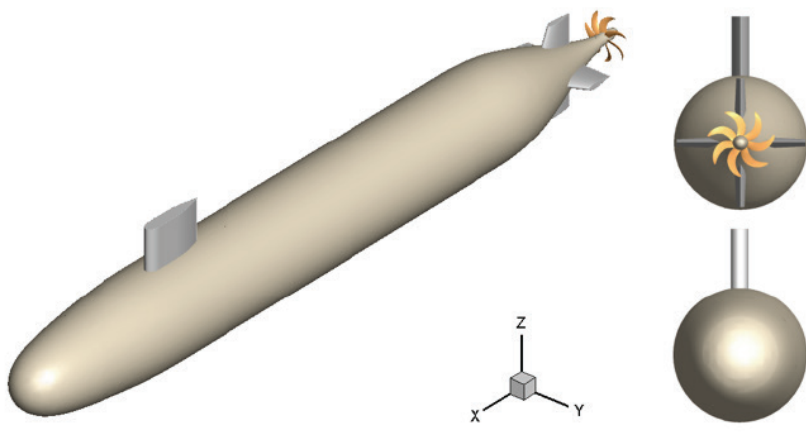

Figure 1. Geometry of the DARPA submarine model DARPA: Defense Advanced Research Projects Agency of 2LBP from the submarine hull's center. These dimensions were selected to be large enough to capture all flow field changes while complying with the ITTC recommendations [26].

A uniform velocity profile was imposed on the solution domain inlet boundary. The study tested seven distinct inflow velocities: $2.75,3.05,5.14,6.10,7.16,8.23$, and $9.15 \mathrm{~m} / \mathrm{s}$, which corresponds to 5.35, 5.93, 10.00, 11.85, 13.92, 16.00, and 17.79 knots, respectively. Pressure outlet boundary condition is used for the outlet boundary, whereas the submarine hull was treated with a no-slip wall condition. The rest of the domain surfaces were treated with a symmetry condition.

\subsection{Grid Structure}

The solution domain was constructed using unstructured hexahedral elements. Figure 2 depicts the general view of the grid topology. When generating the surface grid, special care was taken to ensure the high curvature of the appendages and propeller blades are well represented. Prismatic layers were used on the solid surfaces along the submarine hull to capture the boundary layer adequately. Positive $y+$ values are kept in the range of $30<y+<300$ on the submarine hull to comply with the RANS closure's standard wall-function approach $\left(y+=u_{\tau} y / v\right.$, where $u_{\tau}$ is the friction velocity, $y$ is the height of the first cell on the wall and vis the kinematic viscosity).

A couple of refinement regions were created on some parts of the solution domain. First, the computational grid was refined around the submarine hull. Furthermore, local grid refinements were applied to the wake region and the appendages for accurate modeling of the possible flow separations and high-velocity gradients. The other refinement was achieved in the propeller slipstream. Additionally, attention is paid to providing a smooth mesh transition and alignment between the inside and outside of the interface boundary around the rotating propeller zone to avoid any other numerical error. The simulations used 2.31 M elements after conducting a grid dependency study.

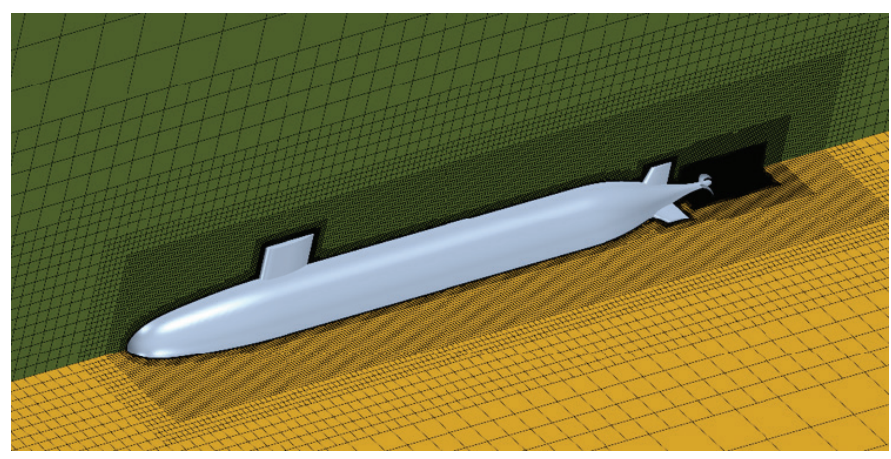

Figure 2. Grid structure around the submarine model 


\section{Numerical Results}

The self-propulsion points, resistance components, and wake structure of the DARPA Suboff at different velocities were determined using CFD. First, a validation of the numerically obtained results was conducted against the related experimental data. As shown in Figure 3, resistance analysis of the DARPA geometry was performed for various velocities and compared with Liu and Huang's [23] experimental measurements. As seen from the figure, numerical results for all data points are in excellent agreement with the experimental results. The maximum relative error between the two sets of results is approximately $2 \%$.

Table 2 presents the self-propulsion characteristics for the DARPA submarine model at $\mathrm{V}=5.35$ knots forward speed and compares it with the results of other studies. In Table 2, Oscillating Water Column (OWC) denotes the open water curve. The calculated thrust coefficient has been placed at the matched point of the propeller's open water performance curve to obtain the corresponding advance coefficient at that self-propulsion point. The thrust and torque coefficients were calculated using the thrust and torque values from the self-propelled CFD analysis. The calculation 3 and 4 are as follows;

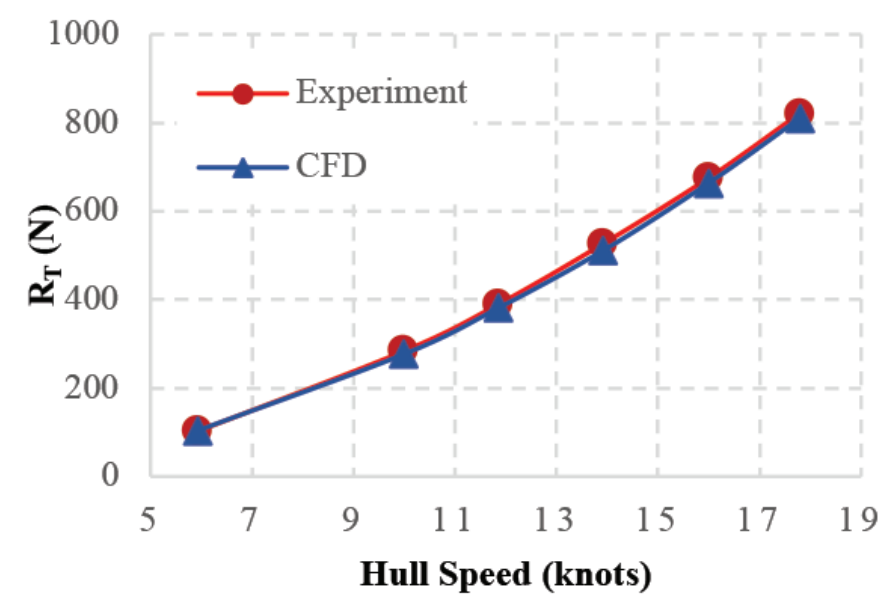

Figure 3. Total resistance calculations for DARPA hull

DARPA: Defense Advanced Research Projects Agency, CFD: Computational fluid dynamics
$K_{T}=\frac{T}{\rho n^{2} D^{4}}$

$K_{Q}=\frac{Q}{\rho n^{2} D^{5}}$

here $T$ and $Q$ denote the trust and torque values, respectively. $\mathrm{D}$ is the propeller diameter. The advance ratio of the propeller is calculated using the axial velocity $V_{A}$ that the propeller receives and the rotation rate $n$;

$$
J=\frac{V_{A}}{n \cdot D}
$$

the open water propeller efficiency is;

$\eta_{0}=\frac{T \cdot V_{A}}{2 \pi \cdot n \cdot Q_{0}}$

here $Q_{\mathrm{O}}$ represents the open water propeller torque. As shown in Table 2, the results of this numerical methodology are close to those of other studies. The advance coefficient and the trust values are remarkably similar to the results presented by Chase and Carrica [24] using experimental OWC. The open water efficiency is within the range of other studies' CFD results, although slightly lower than the experimental OCW predictions. Figure 4 illustrates the DARPA suboff geometry's self-propulsion characteristics determined at higher hull speeds. As shown, the predicted propeller rotation rate values are in good agreement with those of other researchers. The propeller rotational speed (rps- rotation per second) required to drive the hull at a given forward velocity increases with the hull speed. The propeller rotation rates curve at the self-propulsion point has a linear trend. In contrast, the variation of other characteristics to changing hull speed is nearly constant. Similar findings were reported by Kinaci et al. [27]. They numerically investigated the self-propulsion performance of the benchmark DTC hull and showed that $\mathrm{J}_{\mathrm{T}} \mathrm{K}_{\mathrm{T}}$ and $\mathrm{K}_{\mathrm{Q}}$ remain nearly unchanged, whereas the propeller rotation rate increases with increasing Froude Number.

Table 2. Self-propulsion results at $V=5.35$ knots

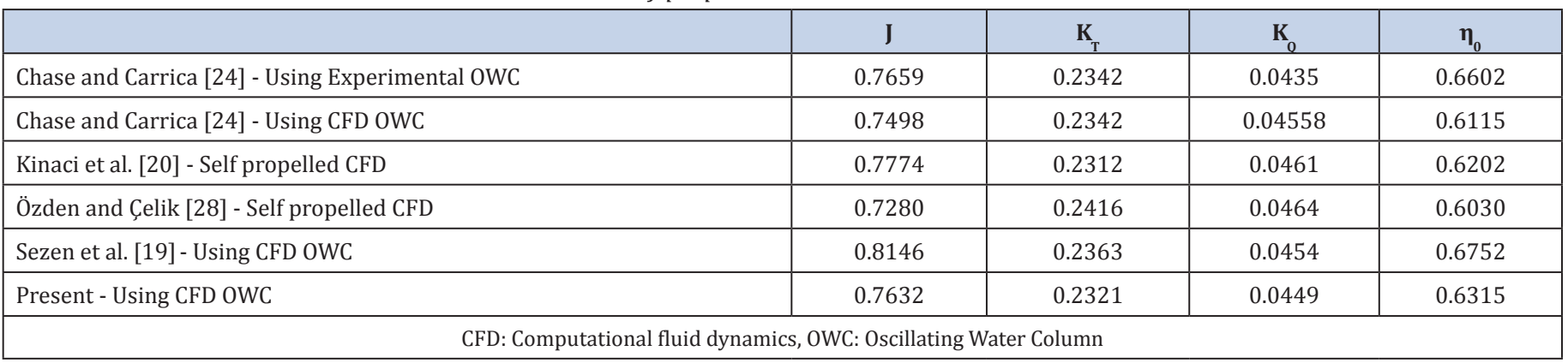



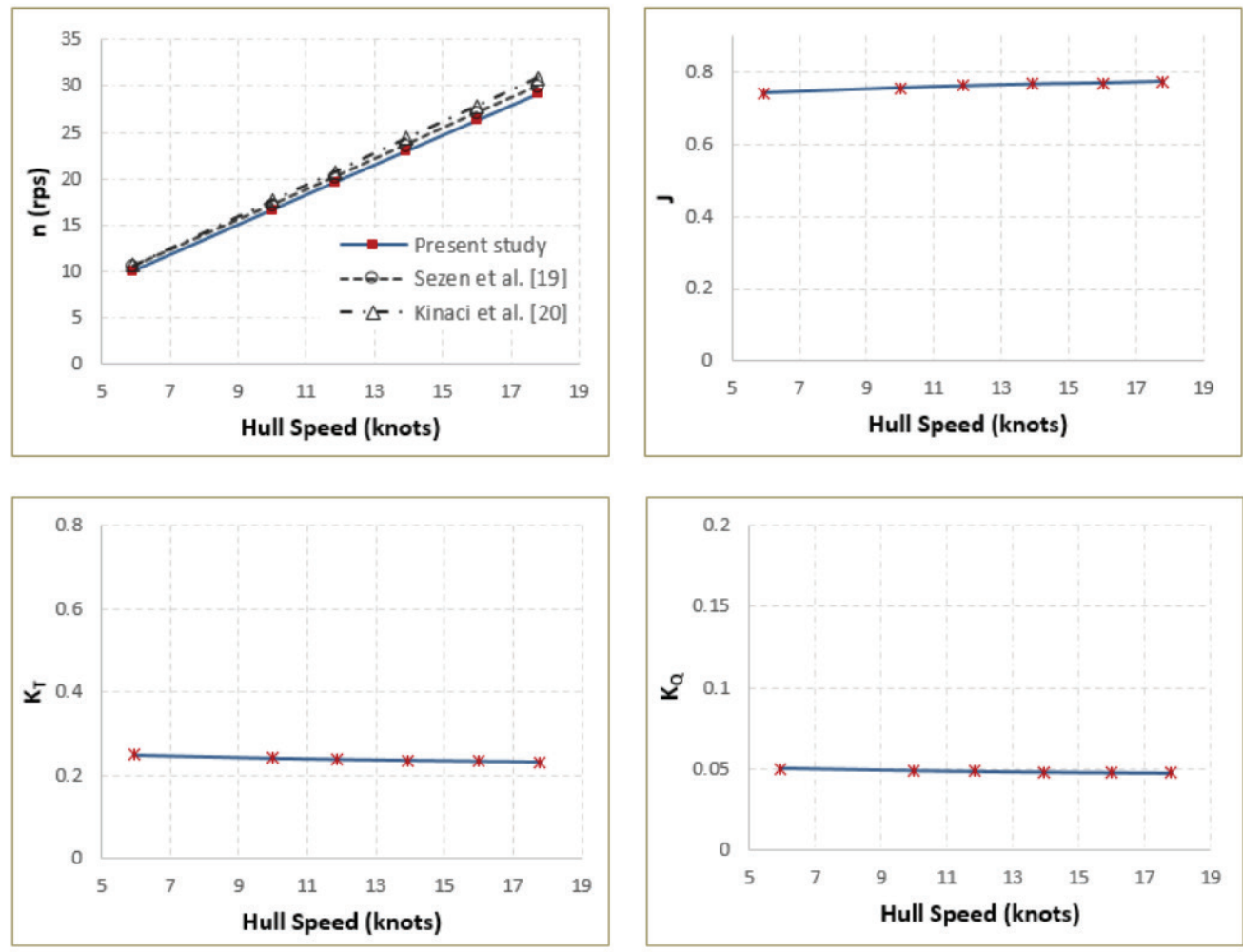

Figure 4. Self-propulsion characteristics of DARPA suboff at different forward velocities

DARPA: Defense Advanced Research Projects Agency

Figure 5 shows the power estimation of the DARPA suboff for determined self-propulsion points. The figure includes the effective power $\left(P_{E}\right)$ that is required to pull the hull at a constant speed and the power delivered to the propeller $\left(P_{D}\right)$. The effective power is computed as follows:

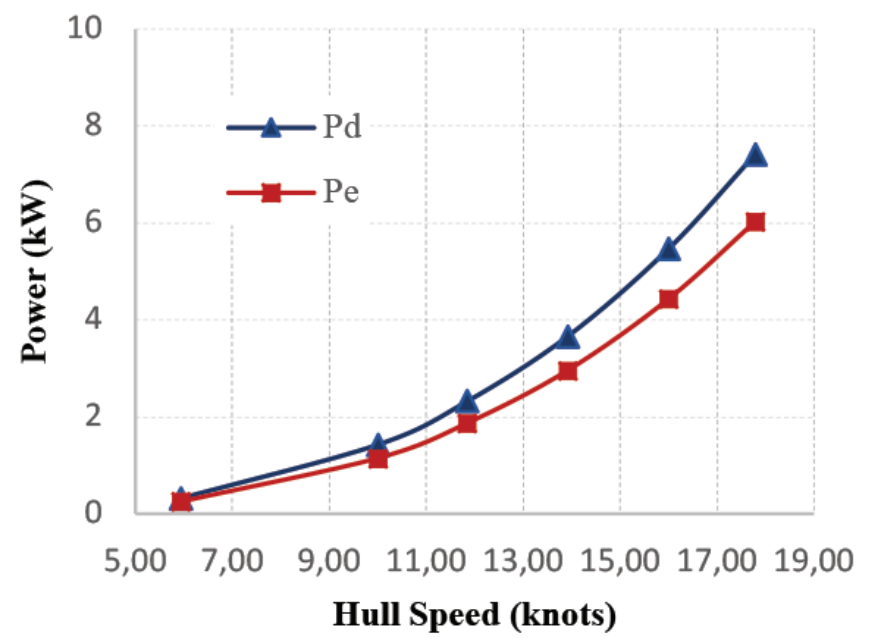

Figure 5. Required power predictions of DARPA hull DARPA: Defense Advanced Research Projects Agency
$P_{E}=R_{T} \cdot V_{S}$

where $R_{T}$ is the total resistance of the hull and $V_{S}$ is the given hull velocity. Then the power delivered to the propeller is calculated as:

$P_{D}=\frac{P_{E}}{\eta_{D}}$

In Equation 8, $\eta_{D}$ represents the propulsion efficiency and can be calculated as: $\eta_{D}=\eta_{H} \cdot \eta_{0} \cdot \eta_{R^{\prime}}$, where the hull efficiency $\eta_{H^{\prime}}$, and the relative rotation efficiency $\eta_{R^{\prime}}$, is:

$\eta_{R}=\frac{Q_{0}}{Q}$

$\eta_{H}=\frac{1-t}{1-w}$

In Eq. 10, the thrust reduction factor $t$ can be obtained using the total towed resistance of the hull and the thrust generated by the propeller using $t=T-R_{T} / T$. The nominal wake coefficient $W$ can be calculated using the axial velocity that the propeller receives and the hull velocity: $w=V_{s}-V_{A} / V_{s}$. Figure 5 shows that at relatively 
lower hull speeds, the effective and delivered power values are very close to each other at the self-propulsion points. As the forward velocity increases, the effective power falls below the delivered power value owing to the propulsion efficiency in Equation 8. In reality, the propulsion efficiency does not deviate significantly for varying hull velocities, but the increasing effective hull resistance and power values for higher forward speeds are responsible for this discrepancy.

The resistance features of the DARPA hull for the towed and self-propelled cases are also investigated, as Figure 6 depicts. As expected, an increase in the hull's forward speed gives rise to the resistance forces. Considering the total resistance $R_{T}$, the towed and the self-propelled submarine results are similar. The disparity in the calculations increases with increasing hull velocity. When we examine the resistance components in the two scenarios, the friction resistance values are almost equal. However, the computed viscous pressure resistance values show an increasing discrepancy with rising hull speed. We can conclude that the difference between the total resistance values of towed and self-propelled cases is owing to the pressure-related forces.

The normalized axial velocities around the submarine hull are presented in Figure 7 to investigate the flow field around the submarine model. The figure shows the upper and the lower bounds of the investigated hull velocity range. The normalized axial velocity value is non-dimensionalized using the hull velocity $u^{*}=u / V_{S}$ and then presented with the threshold value $u^{*} \leq 1$ to examine the boundary layer distribution. For higher and lower forward speeds, the normalized axial velocity distributions around the submarine hull are similar. Notably, these velocity values are the normalized values, and as the vessel speed increase, the dimensional axial velocities rise in value. Owing to the symmetrical geometry of the submarine model, the axial

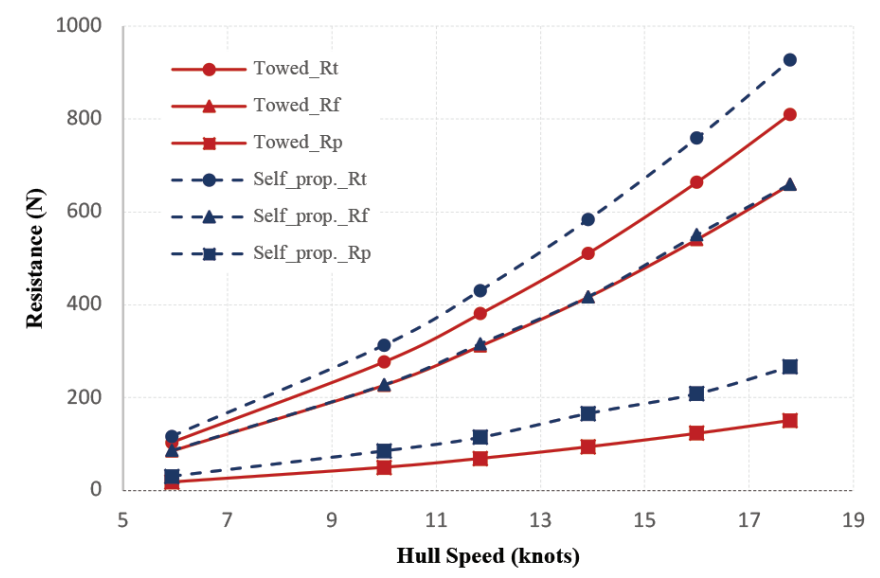

Figure 6. Resistance components of DARPA hull for towed and selfpropelled cases

DARPA: Defense Advanced Research Projects Agency velocity distribution and boundary layer thickness along the hull do not show a strong variation in the streamwise direction, except in the wake region of the sail. The sail's wake seems to vanish toward the aft of the hull. According to Sezen et al. [19] the resistance and self-propulsion analyses of the benchmark DARPA Suboff with E1619 propeller have been done using Computational Fluid Dynamics (CFD, the sail' wake still exists in the propeller plane and slightly accelerates the flow. Compared with the bare form of the DARPA submarine model, they reported that the other appendages also affect the velocity distribution on the propeller plane. Furthermore, Dogrul's [29] revealed that the appendages of the present investigated model had an effect on and raised the resistance and form factor values.

Figure 8 demonstrates the tangential velocity distribution in the propeller's slipstream. The tangential velocity around the submarine hull is mainly concentrated in the propeller slipstream, allowing the propeller wake extension information to be accessible. The tangential velocity values for the lower hull speed are relatively lower and rapidly decreasing with increasing distance from the propeller. As the hull speed increases, the close and far cross-sections tangential velocities from the propeller rises. From Figure 4, the required propeller rotational speed increases to overcome the increasing hull drag for higher forward speeds. As the propeller rotates at higher rps, the propeller wake penetrates through a broader region for high hull speeds. Consquently, a wider wake alters the pressure distribution around the submarine. In contrast to the towed cases, there is no propeller rotation to influence the velocity and pressure distribution on the hull's wake. In the self-propelled cases, a stronger propeller wake with high forward speed possibly causes the discrepancy in the pressure resistance results in Figure 6.
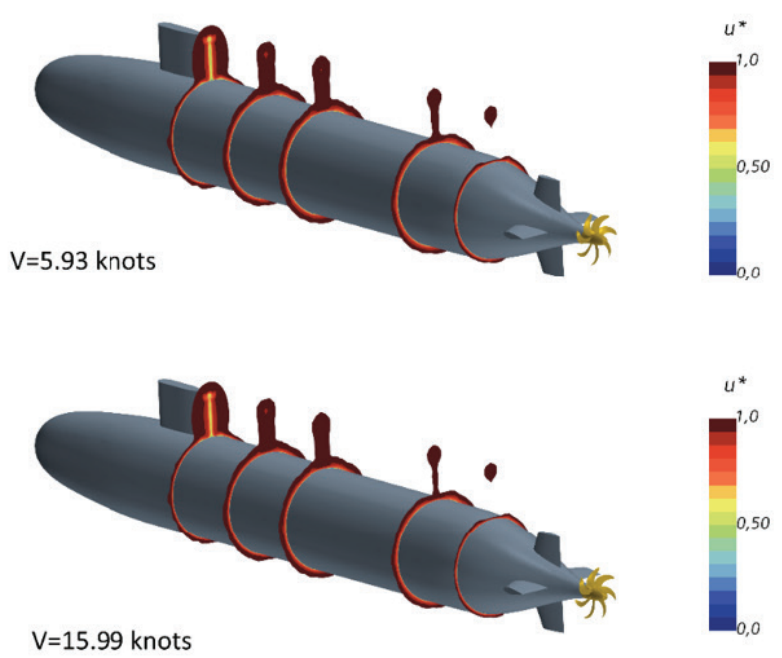

Figure 7. Normalized axial velocities around the hull 


\section{Conclusion}

This study presents the self-propulsion estimation and resistance analysis of the DARPA submarine model. A RANSE based CFD methodology was used to calculate the flow field around the towed and the self-propelled submarine hulls. The propeller rotation for the selfpropulsion computations was modeled using the RBM method. The study aims to assess the submarine model's self-propulsion characteristics for various higher forward speeds and investigate the resistance components variation. The self-propulsion performance of the DARPA suboff for a relatively lower hull speed was calculated and compared to the predictions of other researchers for validation. The results of reported calculations agreed well with that of other studies. Then, using the same methodology, the self-propulsion points for increasing hull forward velocities were estimated. The results reveal that the propeller rotation rate (rps) increases for higher hull speeds, while the advance coefficient (J) remains nearly constant. The power analysis showed a growing disparity between the delivered and the effective powers as the hull speed increased.

The self-propelled hull's total resistance is higher than the towed cases. Considering the resistance components, the frictional resistance values of the two cases are very close; however, the viscous pressure resistance values diverge

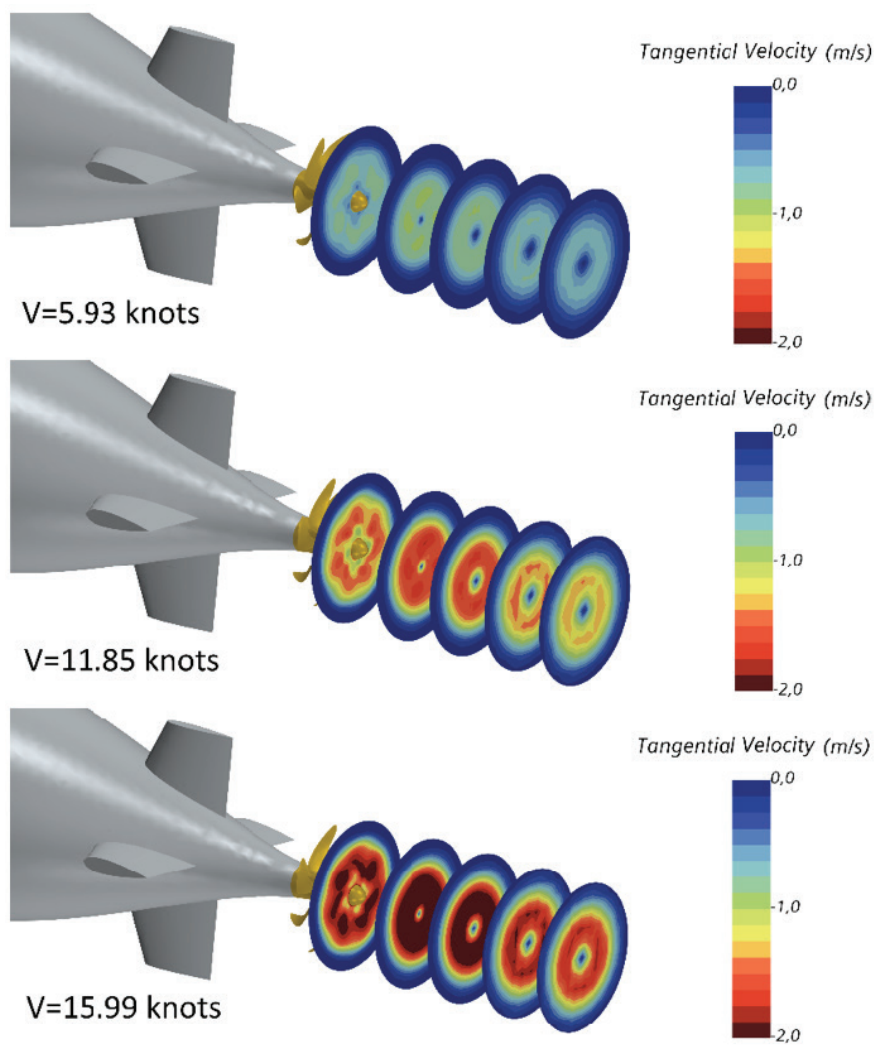

Figure 8. Tangential velocity distributions in the propeller slipstream with increasing hull speed. For the self-propelled cases, the tangential velocity distribution in the propeller slipstream shows a stronger wake with increasing hull speeds. The resistance discrepancies of the towed and self-propelled cases have been attributed to the influence of the propeller rotation on the velocity and pressure distribution on the wake region.

\section{Acknowledgments}

The authors wish to thank Dr. Mario FELLI and INSEAN for sharing the geometry and the experimental data of the INSEAN E1619 submarine propeller.

Funding: The author declared that this study received no financial support.

\section{References}

[1] C. Guo, X. Wang, C. Wang, Q. Zhao, and H. Zhang, "Research on calculation methods of ship model self-propulsion prediction," Ocean Engineering, vol. 203, pp. 107232, May 2020.

[2] L. Zhang, Y. Wei, Y. Deng, H. Yin, Y. Shang and J. Zhang, "A comparative investigation of fixed and free-running CFD selfpropulsion models on a waterjet-propelled trimaran," Ocean Engineering, vol. 232, pp. 109081, Jul 2021.

[3] A.M. Bassam, A. B. Phillips, S. R. Turnock, and P. A. Wilson, "Experimental testing and simulations of an autonomous, self-propulsion and self-measuring tanker ship model," Ocean Engineering, vol. 186, pp. 106065, Aug 2019.

[4] D. Villa, S. Gaggero, T. Gaggero, G. Tani, G. Vernengo, and M. Viviani, "An efficient and robust approach to predict ship selfpropulsion coefficients," Applied Ocean Research, vol. 92, pp. 101862, Nov 2019

[5] F. Jiang, Y. Li, and J. Gong, "Study on the manoeuvre characteristics of a trimaran under different layouts by water-jet self-propulsion model test," Applied Ocean Research, vol. 108, pp. 102550, March 2021.

[6] P.M. Carrica, A. M. Castro, and F. Stern, "Self-propulsion computations using a speed controller and a discretized propeller with dynamic overset grids," Journal Marine Science Technology, vol. 15, pp. 316-330, Dec 2010.

[7] A. M. Castro, P. M. Carrica, and F. Stern, "Full scale self-propulsion computations using discretized propeller for the KRISO container ship KCS," Computers \& Fluids, vol. 51, pp. 35-47, Dec 2011.

[8] Z. Shen, D. Wan, and P. M. Carrica, "Dynamic overset grids in OpenFOAM with application to KCS self-propulsion and maneuvering," Ocean Engineering, vol. 108, pp. 287-306, Nov 2015.

[9] S. Gaggero, D. Villa, and M. Viviani, "An extensive analysis of numerical ship self-propulsion prediction via a coupled BEM/ RANS approach," Applied Ocean Research, vol. 66, pp. 55-78, Jun 2017.

[10] P. He, G. Filip, J.R.R.A. Martins, and K. J. Maki, "Design optimization for self-propulsion of a bulk carrier hull using a discrete adjoint method," Computers \& Fluids, vol. 192, pp. 104259, Oct 2019 . 
[11] A. Bakica, I. Gatin, V. Vukcevic, H. Jasak, and N. Vladimir, "Accurate assessment of ship-propulsion characteristics using CFD," Ocean Engineering, vol. 175, pp. 149-162, Mar 2019.

[12] D. Feng, J. Yu, R. He, Z. Zhang, and X. Wang, "Improved body force propulsion model for ship propeller simulation," Applied Ocean Research, vol. 104, pp. 102328, Nov 2020.

[13] S. Sezen, C. Delen, A. Dogrul, and M. Atlar, "An investigation of scale effects on the self-propulsion characteristics of a submarine," Applied Ocean Research, vol. 113, pp. 102728, Aug 2021.

[14] C. Delen, U. Can, and S. Bal, "Prediction of Resistance and SelfPropulsion Characteristics of a Full-Scale Naval Ship by CFDBased GEOSIM Method," Journal of Ship Research, pp. 1-16, Oct. 2020.

[15] W. Sun, Q. Hu, S. Hu, J. Su, J. Xu, J. Wei and G. Huang, "Numerical Analysis of Full-Scale Ship Self-Propulsion Performance with Direct Comparison to Statistical Sea Trail Results," Journal Marine Science Engineering, vol. 8, pp. 24, Jan 2020.

[16] H. Jasak, V. Vukcevic, I. Gatin, and I. Lalovic, "CFD validation and grid sensitivity studies of full scale ship self propulsion," International Journal of Naval Architecture Ocean Engineering, vol. 11, pp. 33-43, Jan 2019.

[17] N. Chase, Simulations of the DARPA suboff submarine including self-propulsion with the E1619 propeller, İowa: University of Iowa, 2012.

[18] N. Zhang and S.L. Zhang, "Numerical simulation of hull/ propeller interaction of submarine in submergence and near surface conditions," Journal of Hydrodynamics vol. 26, pp. 50-56, Feb 2014.

[19] S. Sezen, A. Dogrul, C. Delen, and S. Bal, "Investigation of self-propulsion of DARPA suboff by RANS method," Ocean Engineering, vol. 150, pp. 258-271, Feb 2018.
[20] O.K. Kinaci, M.K. Gokce, A.D. Alkan, and A. Kukner, "On selfpropulsion assessment of marine vehicles," Brodogradnja, vol. 69, pp. 29-51, Dec 2018.

[21] P.M. Carrica, Y. Kim, and J.E. Martin, "Near-surface self propulsion of a generic submarine in calm water and waves," Ocean Engineering, vol. 183, pp. 87-105, Jul 2019.

[22] N.C. Groves, T.T. Huang, and M. S. Chang, "Geometric characteristics of DARPA suboff models (DTRC model nos. 5470 and 5471) (No. DTRC/SHD-1298-01)," 1989.

[23] H.L. Liu and T.T. Huang, "Summary of DARPA suboff experimental program data (No. CRDKNSWC/HD-1298-11)," Final Rep. Jun 1998.

[24] N. Chase and P.M. Carrica, "Submarine propeller computations and application to self-propulsion of DARPA Suboff," Ocean Engineering, vol. 60, pp. 68-80, Mar 2013.

[25] Siemens, "Star-CCM+ User Guide version 14.02.010" 2019.

[26] ITTC, "Recommended Procedures and Guidelines Practical Guidelines for Ship CFD Applications," 2011. Available from: https://www.scribd.com/document/177879513/ITTC

[27] O.K. Kinaci, M.K. Gokce, and C. Delen, "Resistance experiments and self-propulsion estimations of Duisburg Test Case at 1/100 scale," Ship Technology Research, vol. 67, pp. 109-120, May 2020.

[28] Y.A. Özden and F. Çelik, "Numerical investigation of the effects of underwater aft cone angle and length-to-beam ratio on hull efficiency," Gemi ve Deniz Teknolojisi, pp. 71-88, Jul 2017.

[29] A. Doğrul, "Hydrodynamic investigation of a submarine moving under free surface," Journal of Eta Maritime Science, vol. 7, pp. 212-227, 2019. 